\title{
Embedding damage detection algorithms in a wireless sensing unit for operational power efficiency
}

\author{
Jerome Peter Lynch ${ }^{1,5}$, Arvind Sundararajan ${ }^{2}$, Kincho H Law ${ }^{3}$, \\ Anne S Kiremidjian ${ }^{3}$ and Ed Carryer $^{4}$ \\ ${ }^{1}$ Department of Civil and Environmental Engineering, University of Michigan, \\ 2328 G G Brown, Ann Arbor, MI 48109-2125, USA \\ 2 Department of Electrical Engineering, Stanford University, Stanford, CA 94305, USA \\ ${ }^{3}$ Department of Civil and Environmental Engineering, Stanford University, Stanford, \\ CA 94305, USA \\ ${ }^{4}$ Department of Mechanical Engineering, Stanford University, Stanford, CA 94305, USA \\ E-mail: jerlynch@umich.edu
}

Received 6 June 2003, in final form 3 April 2004

Published 1 June 2004

Online at stacks.iop.org/SMS/13/800

doi:10.1088/0964-1726/13/4/018

\begin{abstract}
A low-cost wireless sensing unit is designed and fabricated for deployment as the building block of wireless structural health monitoring systems. Finite operational lives of portable power supplies, such as batteries, necessitate optimization of the wireless sensing unit design to attain overall energy efficiency. This is in conflict with the need for wireless radios that have far-reaching communication ranges that require significant amounts of power. As a result, a penalty is incurred by transmitting raw time-history records using scarce system resources such as battery power and bandwidth. Alternatively, a computational core that can accommodate local processing of data is designed and implemented in the wireless sensing unit. The role of the computational core is to perform interrogation tasks of collected raw time-history data and to transmit via the wireless channel the analysis results rather than time-history records. To illustrate the ability of the computational core to execute such embedded engineering analyses, a two-tiered time-series damage detection algorithm is implemented as an example. Using a lumped-mass laboratory structure, local execution of the embedded damage detection method is shown to save energy by avoiding utilization of the wireless channel to transmit raw time-history data.
\end{abstract}

(Some figures in this article are in colour only in the electronic version)

\section{Introduction}

Many benefits can be gained from monitoring the ambient and forced response of civil structures such as buildings, bridges, and dams. For example, determination of a structure's dynamic properties from ambient recorded responses can help engineers to identify structural vulnerabilities to large external disturbances. Recorded response data obtained by

5 Author to whom any correspondence should be addressed. structural monitoring systems during earthquakes have proven helpful in identifying response discontinuities attributable to structural damage [1]. As the structural engineering field progresses towards performance-based design principles, structural monitoring systems can provide extensive empirical data that can be used to refine building codes and improve structural models. Structural monitoring can also provide structure owners with rapid insight into the level of seismic excitation exerted on their structures, identify whether their 
structures are safe for occupants following an earthquake and what can be done to improve structural integrity for long-term risk management [2].

The current California Building Code (CBC), based upon the 1997 Uniform Building Code (UBC), recommends installation of structural monitoring systems in structures situated in zones of high seismic activity. A minimum of three accelerometers is suggested for buildings with dimensions of six or more stories and total floor areas greater than $5500 \mathrm{~m}^{2}$ [3]. In addition to buildings, long-span bridges and dams have been instrumented with monitoring systems to measure their responses to earthquakes. In California, over 900 sensing channels have been installed on 60 longspan bridges by the California Department of Transportation and over 100 dams have been instrumented by the California Division of Safety of Dams [4]. Internationally, structural monitoring systems are also widely used to monitor broad classes of structures.

Today, the cost of installing structural monitoring systems in civil structures can be characterized as high. For example, structural monitoring systems installed in buildings can cost $\$ 5000$ per channel with typical installations using 12 sensing channels resulting in total system costs of over $\$ 60000$ [5]. The expensive nature of structural monitoring systems is a direct result of the high installation and maintenance costs associated with system wires. For example, just the installation of the monitoring system can represent up to $25 \%$ of the total system cost where $75 \%$ of the installation time would be focused solely on the installation of the system wires [6] In outdoor applications such as bridges, potentially harsh environmental conditions necessitate additional efforts to install system cables in weatherproof conduits, thereby raising installation costs further. A direct result of high installation costs is that system end users often elect to install a small number of sensors throughout the structure. Small-scale installations are often found to be insufficient for high-rise structures where structural responses are characterized by high-frequency modes with strong participation factors [5].

In cable-based monitoring systems, sensors embedded in the structure communicate their measurements to centralized data repositories. Sensors have no means to locally process their data; rather, the centralized data server is responsible for the aggregation, storage and processing of all measurement data. With engineering analyses, such as damage detection procedures, ordinarily performed by the centralized data server, the monitoring system can become overburdened with computational tasks if the system is comprised of a large number of sensors and stringent real-time demands are imposed on the analysis.

In response to the cost and performance shortcomings of centralized cable-based structural monitoring systems, advanced embedded system technologies have been explored for adoption in recent years. For example, the use of wireless communications to transfer sensor measurements to a centralized data acquisition server was first illustrated by Straser and Kiremidjian [6]. Their work was instrumental in proving the reliability and cost-effectiveness of wireless communications in lieu of extensive cabling in a structure. More recently, Lynch et al [7] have extended their work to couple computational power in the form of low-cost microcontrollers with wireless sensor nodes. The intended purpose of integrating computational power directly with sensors is to permit localized execution of embedded engineering analyses by sensors. Many benefits can be immediately reaped from a wireless monitoring system with embedded computational power. First, decentralization of computational power permits an efficient infrastructure for parallel processing of data. Second, because wireless radios consume large amounts of battery power, processing data at the sensor and transmitting only the results reduces the quantity of raw time-history data to be wirelessly transmitted. Therefore, limited use of the wireless radio can dramatically improve the overall energy efficiency of the wireless structural monitoring system. Energy efficiency is of primary concern because portable batteries with finite operational durations represent the most likely power source for the system's sensor nodes. Other research teams in both academia and industry are producing wireless sensing networks for a variety of applications [8-11]. However, many of the systems developed are generic and do not address the unique demands of the structural monitoring domain where low-power consumption characteristics need to be balanced by far-reaching communication ranges and sufficient computational capabilities for autonomous data processing.

The objective of this research is to present a stateof-the-art wireless sensing unit that is designed for use as a fundamental building block of energy-efficient wireless structural monitoring systems. The research aims to develop a hardware design that is low-cost, low-power yet functionally comparable to current cable-based structural monitoring systems. The embedded intelligence of the wireless sensing unit is in the form of a sophisticated dual-processor core that can be used for localized data interrogation. Interrogation of data directly at the sensor node reduces the demands on the wireless radio thereby preserving the life span of portable power supplies. To illustrate the strength of the computational core, a realistic engineering analysis is embedded in the sensing unit and executed on raw time-history data obtained from a simple lumped-mass laboratory test structure. The embedded analysis chosen for this study is a two-tiered time-series damage detection procedure based upon a statistical pattern recognition paradigm [12]. Damage detection is chosen in this study for inclusion in the wireless sensing unit core because the autonomous execution of such an analysis by the monitoring system nodes is a first step towards developing a comprehensive structural health monitoring system for civil structures. Energy consumed by the wireless sensor in carrying out the embedded damage detection procedure is compared to the energy needed to transmit a raw time-history record. The paper concludes with a brief discussion of the study results and presents current research efforts to embed a broader class of algorithms in the wireless sensing unit core.

\section{Design of a wireless sensing unit for structural monitoring}

A wireless sensing unit is designed as the cornerstone component of a wireless structural monitoring system for civil structures [13, 14]. At the outset of the design process, functional requirements of the sensing unit are specified that 
reflect the demands of structural monitoring applications. First, low-power consumption characteristics of the wireless sensing unit are sought to ensure long-lasting autonomous operation before battery replacement is required. Furthermore, the range of the wireless communication channel must be on the order of $100 \mathrm{~m}$ to permit sufficient separation of the units in large-scale structural systems. An additional motivation for seeking wireless radios with long range is that they exhibit superior propagation properties within enclosed structures such as buildings and dams. A low-cost wireless sensing unit is sought, thereby encouraging installation of structural monitoring systems defined by high sensor densities.

A modular approach is taken in the design of the wireless sensing unit. Principally, the design of the sensing unit can be divided into three functional modules: sensing interface, computational core, and wireless communications. A modular design approach results in a sensing unit that can easily be upgraded as embedded system technologies continue to mature. In addition, dividing the sensing unit design into functional categories favors optimization of each module with respect to cost, desired functionalities, and power consumption characteristics.

\subsection{Sensing interface}

A plethora of sensors can be used to measure the environmental loading and response of structural systems. The wireless sensing unit should be capable of permitting easy interface of traditional sensors such as accelerometers and strain gages as well as new sensors potentially relevant to structural monitoring applications. A sensor transparent interface is designed with multiple channels to accommodate sensors with both analog and digital outputs. Such a multi-channel interface supports multi-sensor data fusion where the outputs of some sensors are used to attain accurate calibration or enhancement of another [15].

The key hardware component of the sensor interface is a single-channel, 16-bit analog-to-digital (A/D) converter that is chosen to accommodate external sensors with analog outputs. With a maximum sampling rate of $100 \mathrm{kHz}$, the Texas Instruments ADS7821 A/D converter can even be used to collect the local response of structural members whose dynamics are defined by high-frequency modes. At the maximum sampling rate of $100 \mathrm{kHz}$, the converter draws $16 \mathrm{~mA}$ of current. For lower sampling rates $(20-200 \mathrm{~Hz})$, the current draw of the A/D converter will be on the order of $1 \mathrm{~mA}$. Two additional sensor channels are provided by the interface for external sensors with digital outputs. In total, three sensor channels are provided by the sensing interface.

\subsection{Computational core}

The most important component of the proposed wireless sensing unit design is the computational core. Core responsibilities include overall operation of the wireless sensing unit in addition to the processing of acquired time-history data. The core is comprised of embedded microcontrollers and their appropriate support circuitry. Commercial microcontrollers come in different sizes (internal bus size), speeds and costs. Low-power microcontrollers tend to be found in eight-bit architectures. While such microcontrollers could easily accommodate the operation of the sensing unit, computationally intense engineering analyses embedded in the core would be difficult to implement. To address the need for high analysis throughput, higher-end microcontrollers, namely 32-bit architectures, are required. Unfortunately, the power consumption characteristics of 32bit microcontrollers exceed design requirements and would drain portable power supplies rapidly. A balance can be attained by designing a computational core with two processors: a low-power eight-bit microcontroller for overall unit operations and a 32-bit microcontroller for execution of embedded engineering analyses. Normal operation of the wireless sensing unit would rely upon the eight-bit microcontroller. When data are ready for processing, the eightbit microcontroller would turn the 32-bit microcontroller on and command it to interrogate the data. Upon completion of the prescribed analyses, the eight-bit microcontroller will record the results and turn the 32-bit microcontroller off. This master-slave relationship exploits the strengths of the two microcontrollers, resulting in an overall low-power core without sacrificing computational capabilities.

A low-power eight-bit microcontroller is selected to control the data acquisition operation of the wireless sensing unit. Specifically, the Atmel AVR AT90S8515 microcontroller is chosen [16]. By leveraging the internal services provided by the AT90S8515, reliable acquisition of sensor data from the sensing interface can be performed in real time. The wireless communication channel is directly accessed through the AT90S8515's serial port. With only $8 \mathrm{kB}$ of read-only memory (ROM), $512 \mathrm{~B}$ of random access memory (RAM) and limited computational speeds available on the AT90S8515, a second microcontroller is selected for inclusion in the computational core. The Motorola MPC555 PowerPC, a highperformance 32-bit microcontroller, is selected for the task of local data interrogation [17]. With $448 \mathrm{kB}$ of ROM and $26 \mathrm{kB}$ of RAM onboard, in addition to a faster clock rate of $20 \mathrm{MHz}$, intensive data processing not possible on the AT90S8515 can now be performed with ease by the PowerPC. The AT90S8515 (at $4 \mathrm{MHz}$ ) draws $8 \mathrm{~mA}$ of current when turned on and active while the MPC555 (at $20 \mathrm{MHz}$ ) draws $110 \mathrm{~mA}$. When placed in sleep mode, both microcontrollers draw reduced currents of 2.5 and $4 \mathrm{~mA}$ for the AT90S8515 and MPC555 respectively.

When data are collected from an externally interfaced sensor, the measurement data can be stored in two memory locations. A static random access memory (SRAM) chip is added to the computational core to hold long time-history records. A Hitachi HM628512B SRAM chip, with a $512 \mathrm{kB}$ capacity, is selected for this purpose because of its rapid read and write times, as well as its low-power-consumption characteristics. For shorter time-history records, the internal RAM of both the Atmel AVR and Motorola PowerPC microcontrollers can be used.

\subsection{Wireless communications}

In exchange for reliable cable-based communications, a lowcost and flexible wireless communication system is chosen. For installation in civil structures, wireless communication components must have node to node ranges of over $150 \mathrm{~m}$ and employ spread spectrum techniques to ensure reliability 
in the face of channel interference, multi-path reflection, and path losses. Furthermore, wireless communication requires adequate penetration characteristics through typical civil engineering materials such as heavily reinforced concrete [18].

The Proxim RangeLAN2 7911 radio modem is selected to serve as the wireless technology for the sensing unit. Operating on the $2.4 \mathrm{GHz}$ unregulated FCC industrial, scientific, and medical (ISM) band, the RangeLAN2 communicates at a data rate of $1.6 \mathrm{Mbps}$. A standard RS232 serial port interface is provided by the modem for direct communication with the computational core. By employing a $1 \mathrm{dBi}$ omnidirectional antenna, open space communication ranges of over $300 \mathrm{~m}$ can be attained, which is a suitable range for the installation of sensing units on bridges. However, the shielding behavior of heavy construction (e.g. concrete) could reduce the range to approximately $150 \mathrm{~m}$ when used in the interior of structures. The wireless modem draws $190 \mathrm{~mA}$ of current while actively receiving and transmitting. Compared to the power consumption characteristics of the computational core, the large power demands of the wireless modem provide additional motivation for performing as many data interrogations as possible using the sensing unit.

\subsection{Wireless sensing unit construction and validation}

To house the chosen circuit components, a two-layer printed circuit board is designed and fabricated. The circuit board is designed to keep the form factor of the wireless sensing unit low and to minimize the electrical noise environment. A limitation of the two-layer circuit board design is its inability to sufficiently separate analog and digital circuit components, resulting in low levels of injected noise in the A/D conversion process. As a result, the effective resolution of the conversion is on the order of 13 bits. The loss of $\mathrm{A} / \mathrm{D}$ resolution is regained by the wireless sensing unit by over-sampling the sensor output with consecutive samples averaged to eliminate electrical noise. The wireless sensing unit can be powered by a $6 \mathrm{~V}$ or higher direct current (DC) power supply. In particular, a high-energy-density ( $\mathrm{Li} / \mathrm{FeS}_{2}$ cell chemistry) $7.5 \mathrm{~V}$ battery pack is chosen to power the completed wireless sensing unit. A conservative estimate for the anticipated life expectancy of the wireless sensing unit using this battery pack is approximately 50 total hours; duty cycle usage in the field could extend the $50 \mathrm{~h}$ battery life to the order of a year.

The completed wireless sensing unit, as pictured in figure 1, has been previously tested and its performance validated in both laboratory and field settings. Simple laboratory test structures have been used to successfully collect data from microelectromechanical system (MEMS) accelerometers in addition to locally calculating the frequency response functions of time-history data [7]. The unit has also been taken to the field for instrumentation upon the Alamosa Canyon Bridge in southern New Mexico in parallel to a commercial cable-based data acquisition system. The accuracy of the wireless monitoring system has been shown to be comparable to that of the commercial system. In addition, installation of the wireless sensing units on the bridge was completed in less than half the time required for the cablebased system [19].

\section{Embedded algorithms for local data interogation}

Nearly an infinite number of engineering analyses can be envisioned for embedment within the proposed wireless sensing unit. For instance, data interrogation tasks associated with damage detection, system identification, and structural control can be of primary interest to the structural engineering community. All of the methods mentioned share the common task of processing raw time-history records to extract pertinent results of great interest to the system end user. Currently, these analyses would be carried out by an engineering professional off line and often long after the data was collected. A distinctive characteristic of the wireless sensing unit is its ability to execute in real time embedded engineering analyses. This characteristic is attractive from a functional standpoint because the individual nodes of the wireless monitoring system can interrogate their measurements, resulting in parallel processing of time-history data. As will be shown, an additional attraction of using the wireless sensing units for data processing is the tremendous gains made in the overall system power efficiency. To illustrate, this study will focus upon the implementation of a promising damage detection algorithm to assess the amount of power saved by the wireless sensing unit in locally processing raw time-history records in lieu of transmitting those records to a centralized data server. It should be noted that other embedded analyses could have easily been used to draw similar conclusions.

An extensive body of literature has illustrated the successes and failures of different potential damage detection algorithms that have been applied to a broad class of structural systems [20]. Early damage detection methods that relied upon modal properties and finite element representations of the structural system have been difficult to apply to civil structures because of environmental and operational variability. As a result, statistical time-series approaches that do not require information on the system variability have been proposed for detecting possible damage in civil structures. For example, statistical time-series approaches have been successfully applied to laboratory test structures and the hull of a high-speed patrol boat [21, 22]. The statistical time-series approach has also proven successful in identifying structural damage that is in the vicinity of accelerometers collecting response data of a benchmark steel civil structure during ambient and forced vibrations [23]. An attractive feature of the time-series approach is that it is intended for implementation with measurement data collected from a single node of the dynamic system to detect damage in the vicinity of the measurement point. Without requiring raw time-history data to be exchanged between sensor nodes, the computational self-sufficiency of the wireless sensor is preserved by the analysis methodology.

\subsection{A statistical time-series damage detection algorithm}

By viewing the detection of damage in a structural system as a pattern classification problem, many tools available for statistical pattern recognition can be evaluated and deployed. The success in applying those tools lies in judiciously choosing appropriate indicators of the structural system that exhibit change to damage. From a structural dynamics standpoint, 


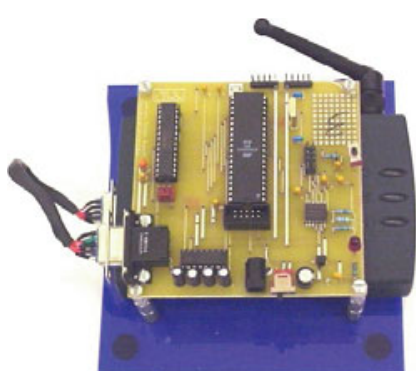

Completed Wireless Sensor Prototype Dimensions $-10 \mathrm{~cm} . \times 10 \mathrm{~cm} \times 3.3 \mathrm{~cm}$

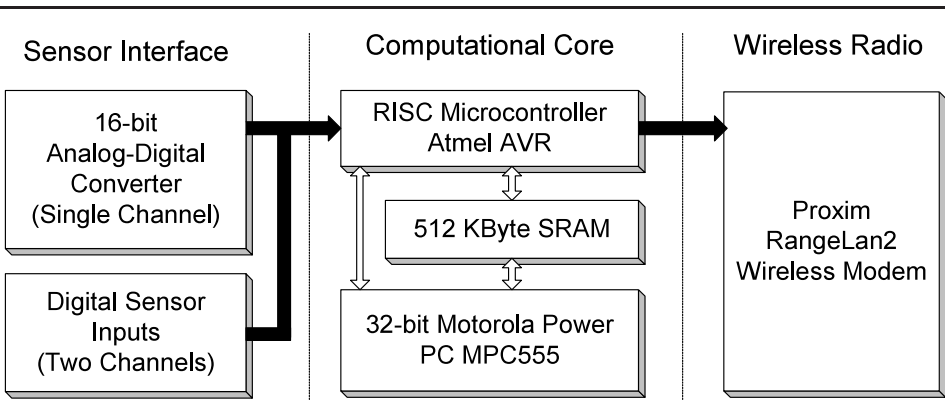

External Power Source - 6V Minimum / 7.5V and 9V Typical

Figure 1. Fully fabricated unit (left) and organizational design (right) of the wireless sensing unit.

time-series models commonly used in system identification studies are natural candidates that exhibit sensitivity to structural damage.

As proposed by Sohn et al [22] the time-series analysis begins with some response measurements, $y$, of the structure at a particular sensor location. Assuming the response to be stationary, an auto-regressive (AR) process model is used to fit the discrete measurement data to a set of linear coefficients weighing past time-history observations:

$$
y_{k}=\sum_{i=1}^{p} b_{i}^{y} y_{k-i}+r_{k}^{y} .
$$

The response of the structure at sample index $k$, as denoted by $y_{k}$, is a function of $p$ previous observations of the system response, plus a residual error term, $r_{k}^{y}$. Weights on the previous observations of $y_{k-i}$ are denoted by the $b_{i}$ coefficients. A large number of AR models can be derived for an undamaged structure under a variety of operational conditions to populate a database consisting of AR model coefficients $\left(b_{i}^{\mathrm{DB}}\right)$. This database is important since it provides a statistical basis for judging whether future AR models represent statistical outliers (and hence indicate possible damage). If the structure is damaged, an AR model fit to time-history data would not be in agreement with the database models that correspond to the undamaged structure. Model agreement, $D$, can be calculated by determining the Euclidian distance between coefficient vectors of the AR model calculated and those in the database. Essentially a nearest-neighbor approach, small distances between coefficient vectors suggest very strong agreement between two time-series models:

$$
D=\sum_{i=1}^{p}\left(b_{i}^{\mathrm{DB}}-b_{i}^{y}\right)^{2} .
$$

It is assumed that the residual error of the AR model, $r_{k}^{y}$, is influenced by the unknown excitation input to the system. As a result, a second time-series model is chosen to model the relationship between the residual error and the measured response of the system. For this second model, an autoregressive with exogenous inputs (ARX) model can be chosen:

$$
y_{k}=\sum_{i=1}^{a} \alpha_{i} y_{k-i}+\sum_{j=0}^{b} \beta_{j} r_{k-j}^{y}+\varepsilon_{k}^{y} .
$$

Coefficients on past measurements and the residual error of the AR model are $\alpha_{i}$ and $\beta_{i}$, respectively. The residual of the ARX model, $\varepsilon_{k}^{y}$, is a damage sensitive feature used to identify the existence of damage in the structure regardless of its operational state. Statistics of the residual error in the ARX model will then be used to hypothesize damage in the structure.

\subsection{Implementation of the two-tiered time-series damage detection algorithm}

To implement the statistical pattern recognition approach, the structure is observed in its undamaged state under a variety of environmental and operational conditions in order to populate a database of AR models of dimension $p$ (denoted as $\mathrm{AR}(p)$ ) paired with ARX models of dimension $a$ and $b$ (denoted as $\operatorname{ARX}(a, b))$. The standard deviation of the residual error of the database ARX model, $\sigma\left(\varepsilon^{\mathrm{DB}}\right)$, is also stored. Prior to using the raw time-history records, the mean and variance of the records are normalized to zero and one respectively. After measuring the response of the structure, $y_{k}$, in an unknown state (damage or undamaged), an $\operatorname{AR}(p)$ model is fitted. The coefficients of the fitted AR model are compared to the database of AR-ARX model pairs previously calculated for the undamaged structure. A match is determined by minimizing the Euclidian distance, $D$, of the newly derived AR model and the database AR model coefficients, $b_{i}^{y}$ and $b_{i}^{\mathrm{DB}}$, respectively. If no structural damage is experienced and the operational conditions of the two models are close to one another, the selected AR model from the database will closely approximate the measured response. If damage has been sustained by the structure, even the closest AR model of the database will not approximate the measured structural response well.

The measured response of the structure in the unknown state, $y_{k}$, and the residual error of the fitted AR model, $r_{k}^{y}$, are substituted into the database ARX model to determine the residual error, $\varepsilon_{k}^{y}$, of the $\mathrm{ARX}$ model:

$$
y_{k}=\sum_{i=1}^{a} \alpha_{i}^{\mathrm{DB}} y_{k-i}+\sum_{j=0}^{b} \beta_{j}^{\mathrm{DB}} r_{k-j}^{y}+\varepsilon_{k}^{y} .
$$

The residual error of the $\operatorname{ARX}(a, b)$ model is the damage sensitive feature in the analysis. If the structure is in a state of damage, the statistics of the ARX model residual, $\varepsilon_{k}^{y}$, will vary from that of the ARX model corresponding to the undamaged structure. In particular, damage can be identified when the ratio of the standard deviation of the model residual error 
Embedding algorithms in a wireless sensing unit for power efficiency

Wireless Sensing Unit

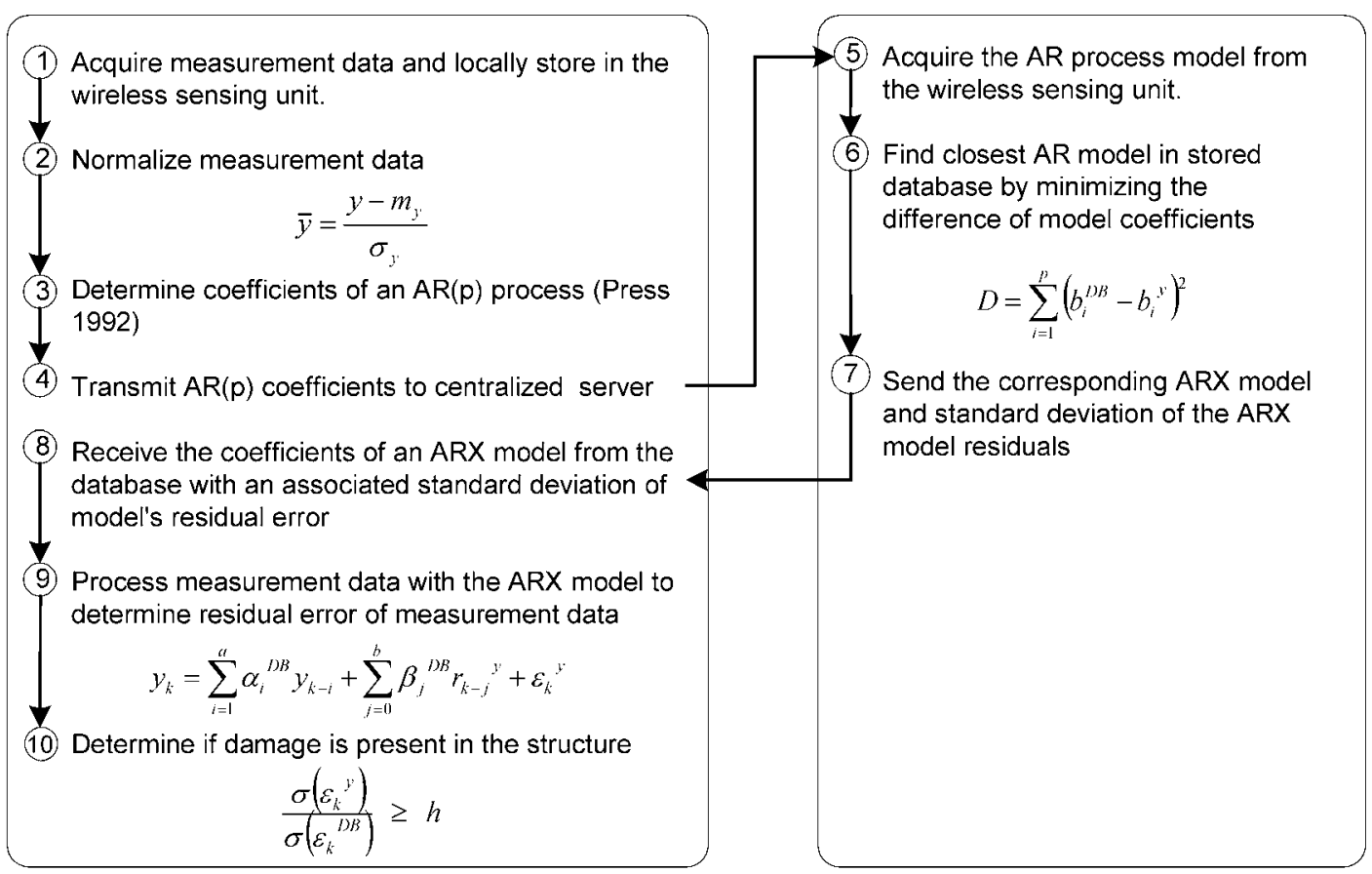

Figure 2. Functional illustration of the autonomous damage detection procedure.

exceeds a threshold value established from good engineering judgment [22].

$$
\frac{\sigma\left(\varepsilon^{y}\right)}{\sigma\left(\varepsilon^{\mathrm{DB}}\right)} \geqslant h .
$$

Establishing a threshold, $h$, that minimizes the number of false-positive and false-negative identifications of damage is necessary for robust damage detection.

The wireless sensing unit will be used to embed the statistical time-series damage detection method presented. Given the memory limitations of the wireless sensing unit, storage of a database of AR and ARX coefficients is done using a remote data server. The implementation details using the wireless sensing unit are presented in figure 2 . The wireless sensing unit is chiefly responsible for the determination of AR model coefficients as well as processing the data through the ARX model that would be obtained from the remote server. The wireless sensing unit, after calculating the ARX residual error, would make the ultimate decision if damage is potentially present in the system within the vicinity of its respective node.

\subsection{Embedded firmware development}

An abstraction layer approach is taken for writing embedded software (also termed firmware) for the wireless sensing unit. The lowest layer of firmware is written to directly interact with hardware subsystems of the sensing unit thereby hiding implementation details from upper software layers. An upper software layer that sits upon the lowest layer is reserved for embedded engineering analyses. At both layers, the unique programming demands of the wireless sensing unit such as limited on-board program and data memory must be addressed to deliver an optimized program.

Software is written to allow the wireless sensing unit to autonomously execute the statistical pattern recognition damage detection method. The code will be responsible for data normalization, determination of statistical means and variances, and the calculation of predicted outputs from timeseries models. However, the most challenging component of the analysis is the code required to determine the AR model coefficients based on a segment of the recorded time-history data. Numerous computational options are available for the determination of AR model coefficients. For example, if the auto-regressive model is written for each of the system's $N$ outputs, a matrix form of the auto-regressive process can be written:

$$
\left[\begin{array}{cccc}
y_{p} & y_{p-1} & \cdots & y_{1} \\
y_{p+1} & y_{p} & \cdots & y_{2} \\
\vdots & \vdots & \ddots & \vdots \\
y_{N-1} & y_{N-2} & \cdots & y_{N-p}
\end{array}\right]\left\{\begin{array}{c}
b_{1} \\
b_{2} \\
\vdots \\
b_{p}
\end{array}\right\}=\left\{\begin{array}{c}
y_{p+1} \\
y_{p+2} \\
\vdots \\
y_{N}
\end{array}\right\} .
$$

Assuming the measurement matrix to be over-determined $(N \gg p)$ and invertible, a least-squares solution to equation (6) could be taken to calculate the coefficients of an AR model. In this study, an alternative approach to determining the AR model coefficients is taken. In particular, Burg's method is selected because it is more stable and ensures greater accuracy compared to the least-squares approach which involves complex matrix inversion operations [24].

The maximum entropy method (MEM) of spectral estimation as proposed by Burg can be used to find an AR time-series model of measurement data equally spaced in time. Burg's method begins by finding a single-coefficient AR model by minimizing the power of the model's prediction error. Subsequent steps find AR models of increasing order, $m+1$, that minimize the prediction error power based on the measurement data and the previously calculated AR model of order $m$ [25]. This process is repeated until an AR model of the desired order, $p$, is achieved. 


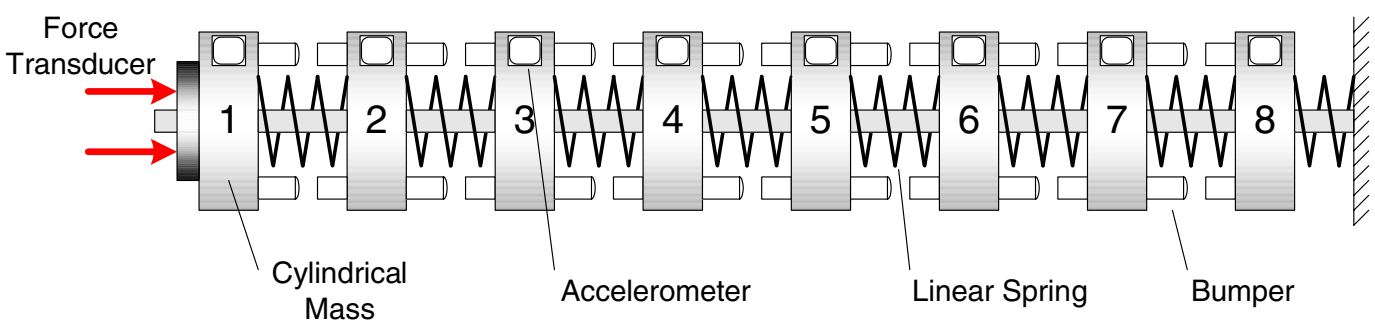

Figure 3. Experimental lumped-mass test structure with damage induced by bumpers between adjacent masses.

\section{Validation of the embedded time-series algorithm}

Raw time-history measurements taken from previous testing of a lumped mass laboratory test structure are used to illustrate the successful execution of the embedded time-series damage detection procedure. The lumped mass model is comprised of eight cylindrical aluminum disks $(25.4 \mathrm{~mm}$ thick, $76.2 \mathrm{~mm}$ diameter) that are free to slide along a common steel rod with coil springs placed between adjacent masses. Each aluminum disk is $419.4 \mathrm{~g}$, except for the first whose mass is $559.3 \mathrm{~g}$, while the spring constants of the coil springs are $56.7 \mathrm{kN} \mathrm{m}^{-1}$. Structural damping is derived from Coulomb damping between the aluminum disks and the steel rod. Endevco 2251A10 accelerometers are firmly attached to each mass of the system to measure transverse acceleration responses from input excitations imparted by a $215 \mathrm{~N}$ electro-dynamic shaker. To induce damage to the structure, adjustable bumpers are placed between masses that make contact when the system is excited by a large force. A picture of the complete test set-up is presented in figure 3 with the aluminum disks numbered.

Sohn and Farrar [12] have extensively used the laboratory test structure to test the proposed time-series damage detection method. In their study, a cable-based laboratory data acquisition system was used to collect the acceleration response of the system to white-noise excitations of prescribed standard deviations. Their time-history records are each 4096 points in length and have been collected at a sampling rate of $512 \mathrm{~Hz}$ and a resolution of 16 bits. The response time-history records collected by their study are stored in their 16-bit digital representations within the wireless sensing unit memory banks for analysis by an embedded damage detection analysis. To simulate the effective 13-bit resolution of the wireless sensing unit A/D converter, Gaussian white noise equivalent to 3 bits of resolution is added to the time-history data.

For both the undamaged (no bumper) and damaged (bumper) states of the structural system, the force transducer is set to exert forces at two operational levels (white-noise forcing functions characterized by standard deviations of 26.6 and $31.1 \mathrm{~N}$ ). Using response data obtained from the undamaged structure, a database of $\operatorname{AR}(30)-\operatorname{ARX}(5,5)$ model pairs is populated. Selection of 30 coefficients for the AR model is determined from the lag value where the autocorrelation function of the response is below a critical threshold near zero. Likewise, the dimensions of the ARX model are chosen to be smaller than the dimension of the AR model as recommended by Ljung [26].

Damage in the structural system is modeled by adjusting the bumper between selected masses to ensure contact during the external excitation [12]. The bumper on the first mass is adjusted to induce contact between mass 1 and 2. The complete two-tiered time-series analysis embedded in the wireless sensing unit is locally executed. Table 1 shows the analysis results, as determined by the wireless sensing unit, with the ratio of standard deviations of equation (5) tabulated. As presented in table 1 , damage is easily identified by the peak in the standard deviation ratio for the data processed in the vicinity of mass 2 . The computational core of the wireless sensing unit has successfully executed the time-series algorithm for detecting the possible existence and location of damage in the system.

\section{Energy efficiencies gained from local data interrogation}

The proposed wireless monitoring system places a strong emphasis upon leveraging the computational strengths of the unit's core to first interrogate data and to then communicate the results to adjacent wireless sensor nodes. Transfer of long records of measured time-history data is not an efficient use of the wireless medium and should be avoided for real-time communication when possible. Results that sufficiently represent the original raw time-history data and that can be communicated to the wireless network include modal frequencies, location and severity of potential structural damage and sensor status information. While transmission of analysis results is preferred from an energy standpoint, raw time-history data can still be locally stored and queried for transmission at a later time.

The rationale for employing the wireless sensing unit's computational core to attain energy efficiency is best explained through the use of an example. Consider an operational scenario where the wireless sensing unit is used to collect a raw time-history record of 4096 points. Since each data point produced by the A/D converter is represented by a 16-bit integer, the resulting record is in total 65536 bits (8192 B). The RangeLAN2 radio is capable of sending data packets with a maximum size of $1462 \mathrm{~B}$ (including $14 \mathrm{~B}$ of overhead per packet). As a result, the entire time-history record can be sent using six packets. At a communication baud rate of 19200 bits per second, the wireless radio requires $4.3 \mathrm{~s}$ of time, $t$, to transmit the data during which time the radio draws $190 \mathrm{~mA}$ of current, $i$. The internal electrical circuit of the wireless radio is regulated at a voltage, $V_{\mathrm{REG}}$, of $5 \mathrm{~V}$. The total amount of energy, $E$, consumed by this operation is then

$$
E=V_{\mathrm{REG}} i t=(5 \mathrm{~V})(0.190 \mathrm{~A})(4.3 \mathrm{~s})=4.09 \mathrm{~J} .
$$

Alternatively, consider the scenario where instead of transmitting the raw time-history record, the MPC555 
Embedding algorithms in a wireless sensing unit for power efficiency

Table 1. Analysis results of the damaged lumped-mass structure (damage induced in the vicinity of mass 2).

\begin{tabular}{lllllllll}
\hline \multirow{2}{*}{$\begin{array}{l}\text { Operational state } \\
\text { Excitation } \sigma(\mathrm{N})\end{array}$} & Mass 1 & Mass 2 & Mass 3 & Mass 4 & Mass 5 & Mass 6 & Mass 7 & Mass 8 \\
\hline 31 & 1.0196 & 2.5181 & 1.3289 & 1.1240 & 1.1170 & 0.9780 & 1.0249 & 1.0401 \\
31 & 1.0034 & 2.4547 & 1.2561 & 1.0320 & 1.0961 & 1.0022 & 1.0116 & 1.0050 \\
31 & 0.9989 & 2.4823 & 1.3454 & 1.0820 & 1.0942 & 0.9799 & 1.0272 & 0.9996 \\
26 & 1.0053 & 2.3187 & 1.2603 & 1.1133 & 1.0876 & 1.0605 & 1.0330 & 1.1117 \\
26 & 1.0039 & 1.9954 & 1.0219 & 0.9573 & 0.9765 & 1.0209 & 0.9873 & 0.9875 \\
26 & 1.0173 & 1.9762 & 1.1441 & 0.9707 & 1.0533 & 1.0463 & 1.0053 & 1.0021 \\
\hline
\end{tabular}

microcontroller is used to execute an engineering analysis and only the results wirelessly transmitted. The MPC555, powered by a direct current power source that is internally regulated at $3.3 \mathrm{~V}$, draws $110 \mathrm{~mA}$ of current. The time required, $t_{\mathrm{MPC} 555}$, for the MPC555 to consume the same amount of energy as that used by the wireless radio in transmitting a 4096-point record is given by

$t_{\mathrm{MPC} 555}=E /(V i)=(4.09 \mathrm{~J}) /(3.3 \mathrm{~V} \times 0.110 \mathrm{~A})=11.27 \mathrm{~s}$.

In this assessment, the amount of energy expended by the wireless radio in transmitting results with sizes of $100 \mathrm{~B}$ or less is negligible. As a result, embedded engineering analyses that can be performed in less than $11.27 \mathrm{~s}$ represent a direct energy saving in the wireless monitoring system.

To empirically determine the energy efficiency of the wireless sensor unit in carrying out the entire statistical pattern recognition damage detection procedure that has been embedded in the wireless sensing unit core, the execution time of the procedure is precisely timed. In particular, steps 2 through 4 and 8 through 10 , as shown in figure 2 , are individually timed. The 32-bit clock of the PowerPC's internal time processing unit (TPU) is employed to serve as a precise means of timing the execution time of each component of the procedure. The execution times measured are required to calculate the amount of battery energy consumed by the PowerPC processor in executing the damage detection analysis. The battery energy consumed by the wireless sensing unit when carrying out the embedded damage detection algorithm will be compared to the energy required to transmit the original raw time-history measurement data to a central data repository.

\subsection{Energy consumed by the wireless transmission of raw time-history data}

The experimental data of the lumped-mass structure is stored within the wireless sensing unit memory bank as floating point numbers. With each measurement point requiring $4 \mathrm{~B}$ of memory, a 4000-point time-history record represents 16000 bytes of stored data. To wirelessly transmit these data using the unit's RangeLAN2 modem, 11 transmission packets are used, each with an overhead of $14 \mathrm{~B}$. In total, $16154 \mathrm{~B}$ are sent to the wireless channel using the serial interface between the computational core and the modem. This transfer of data takes $6.73 \mathrm{~s}$ using the modem's 19200 bits s$^{-1}$ transfer rate. Therefore, the energy consumed by the wireless modem can be determined as follows:

$$
E=V_{\mathrm{REG}} i t=(5 \mathrm{~V})(0.190 \mathrm{~A})(6.7366 \mathrm{~s})=6.400 \mathrm{~J} .
$$

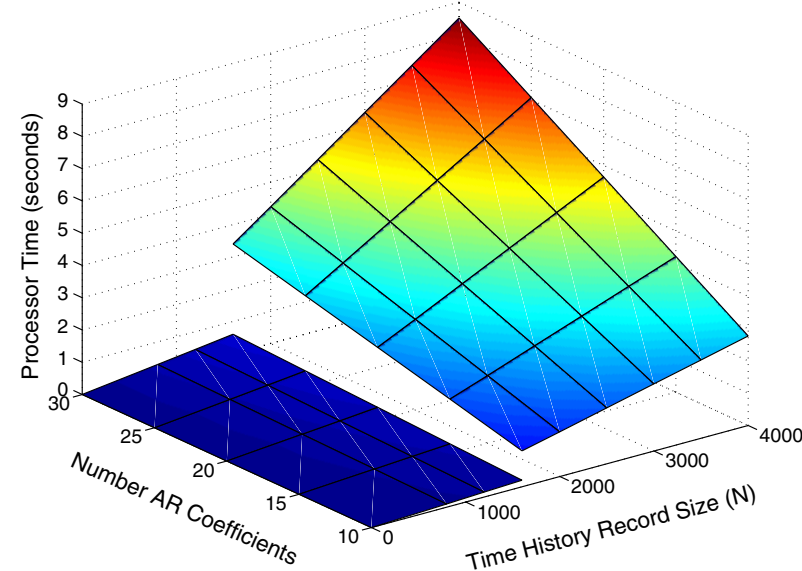

Figure 4. Total time of the PowerPC in determining AR coefficients using the embedded Burgs algorithm (please note the time discontinuity at 1600 points delineating internal and external memory usage).

\subsection{Energy consumed by the local damage detection analysis}

Before an auto-regressive time-series model can be fitted to the experimental data, the data are first normalized to a zero mean and unit standard deviation. The execution time required by the PowerPC to normalize a raw time-history record is a linear function of the number of data points in the record. A time-history record of 1600 points stored in external memory requires $0.0177 \mathrm{~s}$ for the PowerPC to normalize while a record of 4000 points takes $0.0441 \mathrm{~s}$.

Once the time-history record is normalized, an AR timeseries model is fitted to the measurement data. Determination of the AR model coefficients using Burg's method will place the greatest computational demand on the PowerPC processor and will therefore result in the longest compute time compared to the other analysis steps. To observe the dependence of the energy consumed upon the complexity of the algorithm, the number of data points in the time-history record and the number of AR coefficients will both be varied. As shown in figure 5, larger time-history records require more time to calculate a desired number of AR coefficients. Similarly, for a fixed number of time-history data points, a larger number of AR coefficients translates into increases in the processing time. From a time complexity analysis of the coded algorithm, both relationships exhibit linear dependences as observed in the empirical data. For a 4000-sample time-history record, determination of an AR model of 30 coefficients requires $8.351 \mathrm{~s}$ which is orders of magnitude greater than the time needed to normalize the data. 


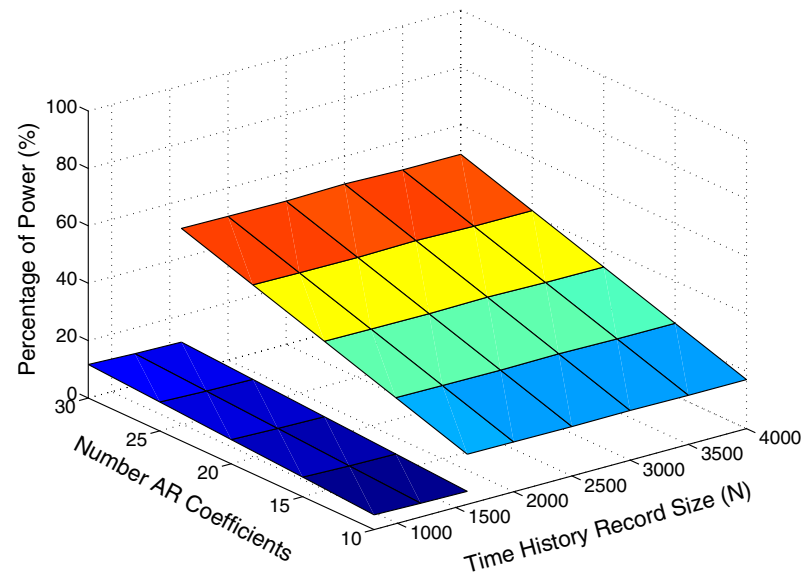

Figure 5. Percentage of energy consumed by the PowerPC in executing the entire damage detection method compared to wirelessly transmitting raw time-history data.

In figure 4, a discontinuity in the time required for execution of Burg's algorithm is witnessed at 1600 data points. This discontinuity results from data being stored in two locations on the wireless sensor unit: in internal and external memory. For shorter time-history records (namely, those smaller than 1600 data points), the internal RAM of the PowerPC microcontroller can be used for storing raw timehistory data during Burg's algorithm. The major advantage of using this memory is that a minimal amount of time is required to read and write data, but its disadvantage is its limited size. For longer time-history records, external memory is required for storage. Using the wireless sensing unit's Hitachi HM628512B SRAM chip that has been incorporated in the computational core, longer time-history records can be stored and processed. A clear disadvantage of the external SRAM is that for every read and write operation approximately four clock cycles are required, thereby adding time overhead to the execution of Burg's embedded algorithm. This is manifested in the significantly larger execution times for time-history records greater than 1600 points.

The time required by the wireless sensing unit in normalizing the time-history data and calculating the AR coefficients is then used to calculate the total energy consumed for steps 2 and 3 of figure 3. Equation (10) presents the energy consumed by the PowerPC in normalizing a timehistory record of 4000 points and subsequently determining 30 AR coefficients

$$
\begin{aligned}
E & =V_{\mathrm{REG}} i t=(3.3 \mathrm{~V})(0.110 \mathrm{~A})(8.351+0.044 \mathrm{~s}) \\
& =3.047 \mathrm{~J} .
\end{aligned}
$$

Once the AR model coefficients have been found by the wireless sensing unit, the computational core encodes the coefficients in a single communication packet for transmission by the wireless modem. Each coefficient is stored as a floating point number and requires $4 \mathrm{~B}$ of memory. The time required by the wireless modem to wirelessly communicate the coefficients using a transfer rate of 19200 bits s$^{-1}$ is a linear function of the number of coefficients. For an AR model of ten coefficients, the RangeLAN2 requires $0.0225 \mathrm{~s}$ to transmit. However, when 30 AR coefficients are considered, the modem requires $0.0558 \mathrm{~s}$. Once the central data repository has received the AR model, the AR model is compared to a database of AR models of the structure in an undamaged state. The closest model from the database is identified and the AR database model's corresponding ARX model is wirelessly transmitted back to the sensing unit. To receive the ten coefficients of an $\operatorname{ARX}(5,5)$ model, the wireless modem requires $0.0242 \mathrm{~s}$ of operation. Therefore, to complete steps 4 and 8 of the damage detection analysis, the wireless modem will consume $0.076 \mathrm{~J}$ of energy:

$$
\begin{aligned}
E & =V_{\mathrm{REG}} i t=(5 \mathrm{~V})(0.190 \mathrm{~A})(0.0558+0.0242 \mathrm{~s}) \\
& =0.076 \mathrm{~J}
\end{aligned}
$$

In the final stages of the damage detection analysis, the normalized time-history data stored in the wireless sensing unit are applied to the ARX time-series model to determine the model prediction error. Again, the analysis time of the PowerPC is found to be a linear function of the time-history record size. For records of 1600 points, $0.0609 \mathrm{~s}$ are required to determine the model error while records 4000 points in length take $0.1514 \mathrm{~s}$. The standard deviation determined using the model error is calculated in step 10. Again, a linear relationship is found between the energy and the size of the time-history record. Records of 1600 and 4000 points require 0.0136 and $0.0338 \mathrm{~s}$ respectively. In total, the energy consumed during steps 9 and 10 using a 4000-point time-history record is approximately $0.0672 \mathrm{~J}$.

Table 2 summarizes the energy schedule of the wireless sensing unit in determining damage from a time-history record of 4000 points using an ARX model of order $p=30$. As shown, the total energy consumed by the wireless sensing unit in locally executing the embedded damage detection algorithm is approximately $3.19 \mathrm{~J}$. Requiring $3.031 \mathrm{~J}$ of energy, determination of the AR model coefficients by Burg's method consumes $95 \%$ of the total energy required for the analysis. None the less, the $3.19 \mathrm{~J}$ of energy needed to execute the embedded analysis is slightly less than $50 \%$ of the $6.4 \mathrm{~J}$ of energy consumed by the wireless sensing unit in transmitting the raw time-history record to a central data repository without performing any local data interrogation. This serves as an illustration of the energy efficiencies associated with locally processing raw time-history data in lieu of its wireless transmission. In this case, a $50 \%$ saving in energy can be reaped.

Figure 5 presents the total energy consumed by the PowerPC in executing the damage detection method as a percentage of the energy required for transmission of the data using the wireless modem. With Burg's method controlling the energy consumed, the energy analysis is considered for various AR model sizes and record lengths. As shown, significant gains in energy efficiency of the wireless structural health monitoring system are gained by local processing of measurement data. However, the energy saved by localized data interrogation is a function of the number of AR coefficients desired and not of the length of the time-history record. This is because increased energy consumption during interrogation of larger time-history records is cancelled by the increased energy needed to wirelessly transmit the record. 
Embedding algorithms in a wireless sensing unit for power efficiency

Table 2. Summary of energy consumed by the wireless sensing unit in executing the embedded damage detection analysis on a 4000-point time-history record.

\begin{tabular}{llll}
\hline Analysis step & Component used & $\begin{array}{l}\text { Execution time } \\
(\mathrm{s})\end{array}$ & $\begin{array}{l}\text { Power consumed } \\
(\mathrm{J})\end{array}$ \\
\hline 2-normalization & PowerPC & 0.044 & 0.016 \\
3-AR model fitting & PowerPC & 8.351 & 3.031 \\
4-send AR coefficients & RangeLAN2 & 0.056 & 0.053 \\
8-receive ARX model & RangeLAN2 & 0.024 & 0.023 \\
9-process ARX model & PowerPC & 0.151 & 0.055 \\
10-find model error STD & PowerPC & 0.034 & 0.012 \\
Total & - & 8.660 & 3.190 \\
\hline
\end{tabular}

\section{Conclusions}

The development of a wireless sensing unit for deployment in future structural monitoring systems is presented. A major innovation of the proposed unit is the convergence of wireless communications and embedded microcontrollers with sensors. Wireless communications eradicate a need for expensive cabling in a structure while microcontrollers facilitate localized processing of raw time-history data prior to transmission in the wireless network. Distributing computational power throughout the sensor network in this manner attains higher energy efficiency, thereby preserving portable battery operational lives.

This study has focused upon illustrating the performance of the wireless sensing unit computational core by embedding a promising approach to the damage detection problem: statistical pattern recognition damage detection using AR and ARX time-series models. Details unique to implementation in the limited resource microcontroller are addressed. An eightdegree-of-freedom laboratory test structure, whose response data are readily available, is used. The wireless sensing unit is configured for autonomous execution of the embedded damage detection algorithm. With damage introduced in the vicinity of mass 2 , the wireless sensing unit was successful in observing significant elevations in the ratio of the ARX model's residual error standard deviation. The study explored the amount of energy consumed by the wireless sensing unit in carrying out the damage detection analysis. The energy saved by performing the damage detection at the sensor node as compared to wirelessly transmitting the original raw time-history data is shown to be on the order of $50 \%$. Burg's recursive procedure for determining optimal AR model coefficients consumes the majority of the analysis energy. As a result, the size of the AR model should be judiciously selected so as to further reduce the total energy required by the sensing unit, thereby extending the battery life.

Many lessons were learned by performing an energy analysis of the wireless sensing system. End users of the proposed wireless monitoring system must be conscious of the complexities of their embedded analyses with algorithm execution times calculated and empirically verified. For example, it is possible for some exhaustive analyses to consume more energy than that required to wirelessly transmit the data to a remote server for processing. These observations underscore the importance of writing embedded algorithms that have been optimized to perform as rapidly as possible. Another important observation to be made is that analyses that can be performed solely within the internal RAM of the PowerPC processor will be highly desirable as a means of saving energy. With respect to the chosen hardware components of the wireless sensing unit, further energy savings could have been gained by choosing different components with better power consumption characteristics. For example, Min et al [9] have considered the energy attributes of the hardware design of a wireless sensor network for the attainment of a low-power solution.

Plenty of opportunity exists for extending this work to encompass additional embedded algorithms for localized execution in the wireless sensing unit. Previous work has explored embedding fast Fourier transforms in the wireless sensing units to derive the frequency response function of structural systems from raw time-history data. For example, frequency response functions calculated by the wireless sensing unit have been used to estimate the modal frequencies of the Alamosa Canyon Bridge in New Mexico [13]. As the field of damage detection matures, additional damage detection methods can be considered for embedding as they arise.

\section{Acknowledgments}

The lumped mass test data provided by Dr Chuck Farrar and Dr Hoon Sohn of Los Alamos National Laboratory have been invaluable for the progress of this study. Their assistance is greatly appreciated. This research is partially funded by the National Science Foundation under grant numbers CMS9988909 and CMS-0121842.

\section{References}

[1] Bolt B A 2001 Seismic instrumentation of bridges and dams: history and possibilities Proc. Instrumental Systems for Diagnostics of Seismic Response of Bridges and Dams (Richmond, CA: Consortium of Organizations for Strong-Motion Observation Systems) pp 1-2

[2] Savage W U 2001 Private and public strong-motion partnerships Proc. Invited Workshop on Strong-Motion Instrumentation of Buildings (Richmond, CA: Consortium of Organizations for Strong-Motion Observation Systems) pp 57-62

[3] International Conference of Building Officials 20022001 California Building Code-California Code of Regulations title 24, part 2, vol 2 (Whittier, CA: ICBO)

[4] Hipley P 2001 'Caltrans' current state-of-practice Proc. Instrumental Systems for Diagnostics of Seismic Response of Bridges and Dams (Richmond, CA: Consortium of Organizations for Strong-Motion Observation Systems) pp 3-9 
[5] Celebi M 2002 Seismic instrumentation of buildings (with emphasis on federal buildings) Report No. 0-7460-68170 United States Geological Survey (USGS) Menlo Park, CA

[6] Straser E G and Kiremidjian A S 1998 A modular, wireless damage monitoring system for structures Report No. 128 John A Blume Earthquake Engineering Center, Department of Civil and Environmental Engineering, Stanford University, Stanford, CA

[7] Lynch J P, Law K H, Kiremidjian A S, Carryer E, Kenny T W, Partridge A and Sundararajan A 2002 Validation of a wireless modular monitoring system for structures Proc. 9th Annual Int. Symp. on Smart Structures and Materials (San Diego, CA: SPIE Optical Engineering Press)

[8] Warneke B A and Pister K S J 2002 MEMS for distributed wireless sensor networks Proc. 9th Int. Conf. on Electronics, Circuits and Systems (Dubrovnik: IEEE)

[9] Min R, Bhardwaj M, Cho S-H, Shih E, Sinha A, Wang A and Chandrakasan A 2001 Low-power wireless sensor networks Proc. 14th Int. Conf. on VLSI Design (Bangalore: IEEE)

[10] Horton M, Culler D, Pister K S J, Hill J, Szewczyk R and Woo A 2002 MICA - the commercialization of microsensor motes Sensor Mag. 19 23-8

[11] Microstrain Inc. 2002 Wireless Web Sensor Networks-Fact Sheet (Williston, VT: Microstrain Inc.)

[12] Sohn H and Farrar C R 2001 Damage diagnosis using time-series analysis of vibration signals Smart Mater. Struct. $10446-51$

[13] Lynch J P, Law K H, Kiremidjian A S, Carryer E, Farrar C R, Sohn H, Allen D, Nadler B and Wait J 2002 Design and performance validation of a wireless sensing unit for structural monitoring applications Struct. Eng. Mech. 17 393-408

[14] Lynch J P, Law K H, Kiremidjian A S, Kenny T W and Carryer E 2002 A wireless modular monitoring system for civil structures IMAC 20: Proc. 20th Int. Conf. on Modal Analysis (Los Angeles, CA: Society of Engineering Mechanics) pp 1-6

[15] Hall D L and Llinas J 2001 Handbook of Multi-Sensor Data Fusion (Baton Rouge, LA: CRC Press)

[16] Atmel Corporation 2001 Atmel AVR AT90S8515 Data Sheet-8-bit AVR Microcontroller with $8 K$ Bytes in-system Programmable Flash (San Jose, CA: Atmel Corporation)
[17] Motorola Corporation Inc. 2000 MPC555 User's Manual (Phoenix, AZ: Motorola Corporation)

[18] Davidson A and Hill C 1997 Measurement of building penetration into medium buildings at 900 and $1500 \mathrm{MHz}$ IEEE Trans. Veh. Technol. 46 161-8

[19] Lynch J P, Sundararajan A, Law K H, Kiremidjian A S, Carryer E, Sohn H and Farrar C R 2003 Field validation of a wireless structural monitoring system on the alamosa canyon bridge Proc. 10th Annual Int. Symp. on Smart Structures and Materials (San Diego, CA: SPIE Optical Engineering Press)

[20] Doebling S W, Farrar C R, Prime M B and Shevitz D W 1996 Damage identification and health monitoring of structural and mechanical systems from changes in their vibration characteristics: a literature review Report No. LA-13070-MS Los Alamos National Laboratory, Los Alamos, NM

[21] Farrar C R, Duffey T A, Doebling S W and Nix D 2000 A statistical pattern recognition paradigm for vibration-based structural health monitoring Proc. 2nd Int. Workshop on Structural Health Monitoring (Stanford, CA: Stanford University)

[22] Sohn H, Farrar C R, Hunter N and Worden K 2001 Applying the LANL statistical pattern recognition paradigm for structural health monitoring to data from a surface-effect fast patrol boat Report No. LA-13761-MS Los Alamos National Laboratory, Los Alamos, NM

[23] Lei Y, Kiremidjian A S, Nair K K, Lynch J P, Law K H, Kenny T W, Carryer E and Kottapalli A 2003 Statistical damage detection using time-series analysis on a structural health monitoring benchmark problem Proc. 9th Int. Conf. on Applications of Statistics and Probability in Civil Engineering (San Francisco, CA: Civil Engineering Risk and Reliability Association (CERRA))

[24] Burg J P 1978 A new analysis technique for time-series data, NATO Advanced Study Institute on Signal Processing with Emphasis on Underwater Acoustics Modern Spectrum Analysis ed D F Childers (NY: IEEE Press) pp 42-8

[25] Andersen N 1974 On the calculation of filter coefficients for maximum entropy spectral analysis Geophysics 39 69-72

[26] Ljung L 1999 System Identification-Theory for the User 2nd edn (Upper Saddle River, NJ: Prentice-Hall) 